SULLE VAHIAZIONI DIURNE DELLA RIFRAZIONE ATMOSFERIGA

pel Prof. VITTORIO E. BOCCARA ${ }^{1}$ ).

(Targo sunto dell'Autore).

Sin dal 1890 il Prof. Riccò aveva fatto notare che la linea dell' orizzonte inarino cambia sensibilmente di posizione rispetto alle cime che incontra degli edifici della città; tale rariazione è dipendente, come è noto, dalla rifrazione atmosferica per cui si puó, conoscendo quella, misurare la variazione di questa.

Quindi con processo semplice e facile è possibile fare uno studio sulla variazione diurna della rifrazione. Altrj metodi invero vi sarebbero per fare simili studi, ma questi hanno il Ioro fondamento sulla determinazione di distanze zenitali contemporanee, per le quali determinazioni occorrono strumenti di grande precisione che non sono alla portata di tuttj.

Col metodo semplice, a cui alludero più sopra, si puó conoscere l'angolo che una certa visuale diretta all' orizzonte marino fa con una visuale diretta ad un punto fisso, ed ecco come : Coll' aiuto di un cannocchiale si miri la linea dell' orizzonte marino e nel contempo si osservi un qualunque punto la di cui posizione, per la piccola distanza dall' osservatore, si possa ritenere come sensibilmente non variabile. La scelta del punto sarà fatta opportunamente per modo che esso e la sua immagine giacciano sul piano verticale passante per l'asse ottico, orizzontale, del cannocchiale. Collocato in modo invariabile il cannocchiale si osservi più spesso che sia possibile (ogni mezz' ora, come fu fatto in questo primo studio) la linea dell'orizzonte marino e si vedrà che questa cambia sensibilmente di posizione rispetto al punto di riferimento. Si misuri l'angolo che la visuale diretta all' orizzonte e giacente nel piano

1) Lavoro eseguito nell' Osservatorio astrofisico di Catania per gentile concessione del Direttore sig. Prof. Riccò al quale l'A. esprime la sui riconoscenza e pubblicato nelle Memorie della Società degli Spettroscopisti Italiani, VoI. 30, 1901, pag. 162 a pag. 178. 
verticale sumenzionato forma colla visuale diretta al punto di riferimento. Cognito tale angolo, che per brevita diremo $\alpha$, si conoscerà subito la rifrazione.

Avendo io adoperato il cannocchiale o micrometro a doppia jmmagine del Rochon potei direttamente avere gli angoli $a$ con una semplice lettura della divisione e parte di divisione indicate dall' indice del nonio, scorrevole lungo la scala incisa sullo strumento.

Peró per avere resultati esatti il più possibile con tale strumento bisognò che replicassi le osservazioni e prendendo $x$ come medio di dodici consecutive osservazioni ottenevo una approssimazione di circa $\pm 0,05$. Tra un gruppo di osservazioni e l'altro, per riposare anche l' occhio, osservai lo stato e colore del cielo e del mare notandolo nel registro delle osservazioni.

Tenni conto anche dell' ondulazione dell' aria perchè ritenevo che dovesse avere una sensibile influenza sulla rifrazione. Tale ondulazione dipende specialmente dalla diversa temperatura degli strati di aria che dal suolo s' innalzano negli strati superiori. Questa ondulazione è variabile nelle diverse ore del giorno e per poter dare una idea del suo movimento, adottai quella stessa graduazione che adottano all' Osservatorio di Catania per classificare l' agitazione of ondulazione dell'aria al bordo solar'e ${ }^{1}$ ).

Come temperatura dell' orizzonte presi la media tra quella altra volta osservata per il mare Jonio, l'aria sovrastante e la media diurna all' Osservatorio.

Per tutti gli altri dati di temperatura, pressione ecc. mi servii nel modo migliore di quelle che regolarmente venivano fatte dal personale dell' Osservatorio ${ }^{2}$ ).

1) La graduazione si fa con una serio di 10 numeri. Si nota : Ondulazione zero quando l'orlo solare è completamente tranquillo; ondulizione 10 quando l'agitazione è tale che il bordo solare alla sua rolta pare che prenda parte al movímento sì da non puterlo osservare per la dolorosa inpressione the fit sull'nechio. I numeri intermedi indicano movimenti intermedi a quelli accennati. Nelle diverse usservazioni che feci del bordo soiare in compagnia dell assistente Signor Mazzarella presi connscesza di questa scala e me oe servii di poi pel mio caso.

2) Tedi Memoria origituale a pag. 167 a 174 
Cognito l' angolo $\alpha$ per conoscere la depressione rifratta $d_{r}$ dell' orizzonte marino basta aggiungervi la depressione del punto di riferimento. La qual depressione per misure precedentemente fatte dal Prof. Saija con un tacheometro Salmoiraghi risultò di 5,28. Ed io mi servii di questo stesso valore, che non avrei potuto determinare di nuovo per mancanza dello strumento, anche perchè questa costante aggiunta non porta nessuna influenza sulle variazioni della rifrazione. Quindi si avrà

$$
a_{\mathrm{r}}=\alpha+5,28
$$

E noto che la depressione geometrica $d$ dell' orizzonte si calcola colla formola approssimata

$$
\operatorname{tang} d=\sqrt{\frac{2 h}{\mathrm{R}}}
$$

od anche per la piccolezza di $d$ coll' altra

$$
d^{\prime \prime}=\sqrt{\frac{2 h}{\mathrm{R \operatorname {sen } ^ { 2 } 1 ^ { \prime \prime }}}}
$$

la quale nel mio caso per essere $h=\mathrm{m} .63,70$ e $\log$. $\mathrm{R}=$ $=6,805050 \mathrm{dà}$

$$
d=15^{\prime}, 36
$$

valore che si puó ritenere costante, perche l'influenza della variazione di livello dei nostri mari, dovuta alla marea, e stata trovata cosi piccola da essere inferiore agli errori inevitabili di osservazione.

Cognito a si trova pure subito l' angolo di rifrazione, o, come suol dirsi semplicemente, la rifrazione $r$ colla semplicissima relazione

$$
r=a-d_{\mathrm{r}}=15^{\prime}, 36-a_{\mathrm{r}}
$$

e per la (1)

$$
r=10^{\prime}, 08-\alpha .
$$

Dai valori trovati per l'angolo $\alpha$ emerge come la rifrazione sia variabile durante il giorno ed abbia dei massimi, pure variabili, che in generale si presentano intorno alle ore otto antimeridiane. 
Per confrontare la rifrazione osservata $r$ con quella $r_{i}$, che dovrebbe avvenire se l' atmosfera fosse normalmente costituita, calcolo questa servendomi della formola, che lá la depressione tenuto conto della rifrazione,

$$
\mathrm{D}=\sqrt{\frac{2 b}{\mathrm{R}} \cdot \frac{m-1}{m}}=d \sqrt{\frac{m-1}{m}}
$$

nella quale facendo secondo Faye $m=8,2$ e ponendo, come si trovó, $d=15,36$, si avrà

$$
\mathrm{D}=15^{\prime}, 36 \cdot 0,9370=14^{\prime}, 39
$$

e quindi

$$
r_{1}=15^{\prime}, 36-14^{\prime}, 39=0^{\prime}, 97 .
$$

Da tale confronto si vede come la rifrazione osservata sia nel maggior numero dei casi inferiore a quelia normale e questo conferma ancora una volta che in Sicilia la rifrazione è minore di quella osservata in altre regioni.

Dall analisi accurata dei valori giornalmente trovati non si puó stabilire nessuna intima relazione tra la rifrazione e gli elementi meteorici, emerge però : Che diminuisce coll'aumentare della pressione, e col diminuire della differenza di temperatura del luogo di osservazione e quella dell' orizzonte marinn: aumenta col diminuire della pressione e coll'aumentare della sopra detta differenza di temperatura. Ciò è concorde colle ipotesi che comunemente si fanno per spiegare la rifrazione atmosferica.

Nei periodi in cui la pressione e la differenza di temperatura si possono ritenere costanti, la rifrazione è variabile coll' ondulazione dell' aria ed in qualche modo ha un andamento inverso.

Lo stato igrometrico, per quanto leggermente, ha pure la sua influenza, e tende col suo aumento a far aumentare la rifrazione.

Per avere un'idea un poco piu generale sull' andamento diurno della rifrazione trovo la media dei valori notati nella stessa ora che riporto nella tabella seguente: 


\begin{tabular}{|c|c|c|c|c|c|c|c|c|}
\hline \multirow[b]{2}{*}{ ORA } & \multirow{2}{*}{$\begin{array}{l}\text { onduiaz. } \\
\text { aria }\end{array}$} & \multicolumn{2}{|c|}{ Pressione a $0^{0}$} & \multicolumn{2}{|c|}{ Temperatura in $\mathrm{C}$} & \multirow{2}{*}{ 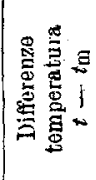 } & \multirow{2}{*}{ Stato } & \multirow[b]{2}{*}{ Rifraz } \\
\hline & & al mare & $\begin{array}{l}\text { al luogo } \\
\text { di } \\
\text { osservaz. }\end{array}$ & al mare & $\begin{array}{c}\text { al luogo } \\
\text { di osserv. } \\
t\end{array}$ & & & \\
\hline 7 & 5 & 762.9 & 757.3 & 14.6 & 13.5 & -1.1 & 52 & $0^{\prime} .98$ \\
\hline 7.30 & 5.5 & 763.5 & 757.0 & 14.4 & 8 & -0.6 & 52 & .77 \\
\hline 8 & 3.2 & 761.9 & 756.3 & 15.4 & 6 & +1.2 & 46 & $\mathrm{l}^{\prime} .39$ \\
\hline 8.30 & 3.4 & 762.0 & 756.4 & 15.5 & .9 & +2.4 & 42 & $1^{\circ} .28$ \\
\hline 9 & 3.4 & 761.4 & 755.8 & 15.6 & & 2.7 & 39 & $1^{\prime} .21$ \\
\hline 9.30 & 3.5 & 761.9 & 756.3 & 15.4 & & 3.0 & 35 & $0^{\prime} .93$ \\
\hline 10 & 4 & 761.4 & $755: 8$ & 4.6 & & 1. & 31 & '. 88 \\
\hline 10.30 & 4.2 & 762.2 & 756.6 & & & 3.4 & 30 & 0.86 \\
\hline 1. & 5 & 76 & & 1 & & 3. & 30 & $0^{\prime} .83$ \\
\hline 11.30 & 5 & 762.2 & 7 & 1 & & 4.6 & 20 & $0^{\prime} .68$ \\
\hline 12 & 5.6 & 762.3 & 75 & 1 & & 4. & 31 & $0^{\prime} .72$ \\
\hline 12.30 & 5.6 & 762.3 & 75 & 7 & & 3. & 31 & $0^{\prime} .70$ \\
\hline 13 & 5.4 & 762.1 & 75 & & & 3.2 & 36 & 69 \\
\hline 13.30 & 5.3 & 76 & $7 !$ & & & 3.1 & 36 & $0^{\prime} .63$ \\
\hline 7 & 5 & & & & & 3. & 40 & $0^{\prime} .67$ \\
\hline 14.30 & 6 & & & & & 2. & 36 & $0^{\prime} .62$ \\
\hline 18 & 4.6 & 762 & 756.7 & 1 & & 2. & 45 & 0.70 \\
\hline 15.30 & 4.6 & 761.4 & 755.8 & & & 3. & 44 & 0.72 \\
\hline 16 & 4.3 & 761.2 & 755.6 & & & 2.7 & 46 & 0.73 \\
\hline 16.30 & 4 & 761 & & & & 2.7 & 53 & 0.74 \\
\hline 0 & 4 & 761.5 & & & & 2.6 & 55 & $0^{\circ} .86$ \\
\hline 17. & 3. & & & & & 22 & 54 & $0^{\prime} .73$ \\
\hline 7 & 2.6 & 76 & & 14.9 & & 2. & 58 & 0.74 \\
\hline
\end{tabular}

e cotruisco le curve rappresentative di tali valori riportando questi sopra le ordinate, corrispondenti ad ascisse indicanti le varie ore del giorno.

Dalla prima curva media (fig. 1) risulta che : La rifrazione è massima verso le 8 ; dopo decresce rapidamente fin verso le $11 \frac{1}{2}$, si mantiene da quest' ora fin verso le ore 15 quasi costante, dopo la qual ora comincia ad aumentare leggermente.

Noto qui che osservazioni prima delle ore 7 e dopo le ore 18 non mi fu possibile fare perchè l' orizzonte marino non $\mathrm{mi}$ appariva netto '7, e che le tre osservazioni delle $13,13,30$ e

1) L' orizzonte prima $\theta$ dopo le ore notate non mi appariva netto per varie ragioni: o non lo potevo fissare per lo splendore del sole da poco solto; o una leggera nebbia I offuscava, o il colore del cielo pareva cosi uguale a quello del unare che non era possibile distinguere la linea di separazione, od infine perchè all' drizzonte apparivan? tante strie lunghe talchè diffeilmente era possibile distinguere quale fosse quella ultima di confiue apparente col cielo. 
VARIAZIONI DIURNE DELLA RIFRAZIONE ATMOSFERICA “ 209

14 furono fatte dall assistente sig. Dott. L. Mendola. Que-

ste osservazioni furono però debitamente corrette avendo,

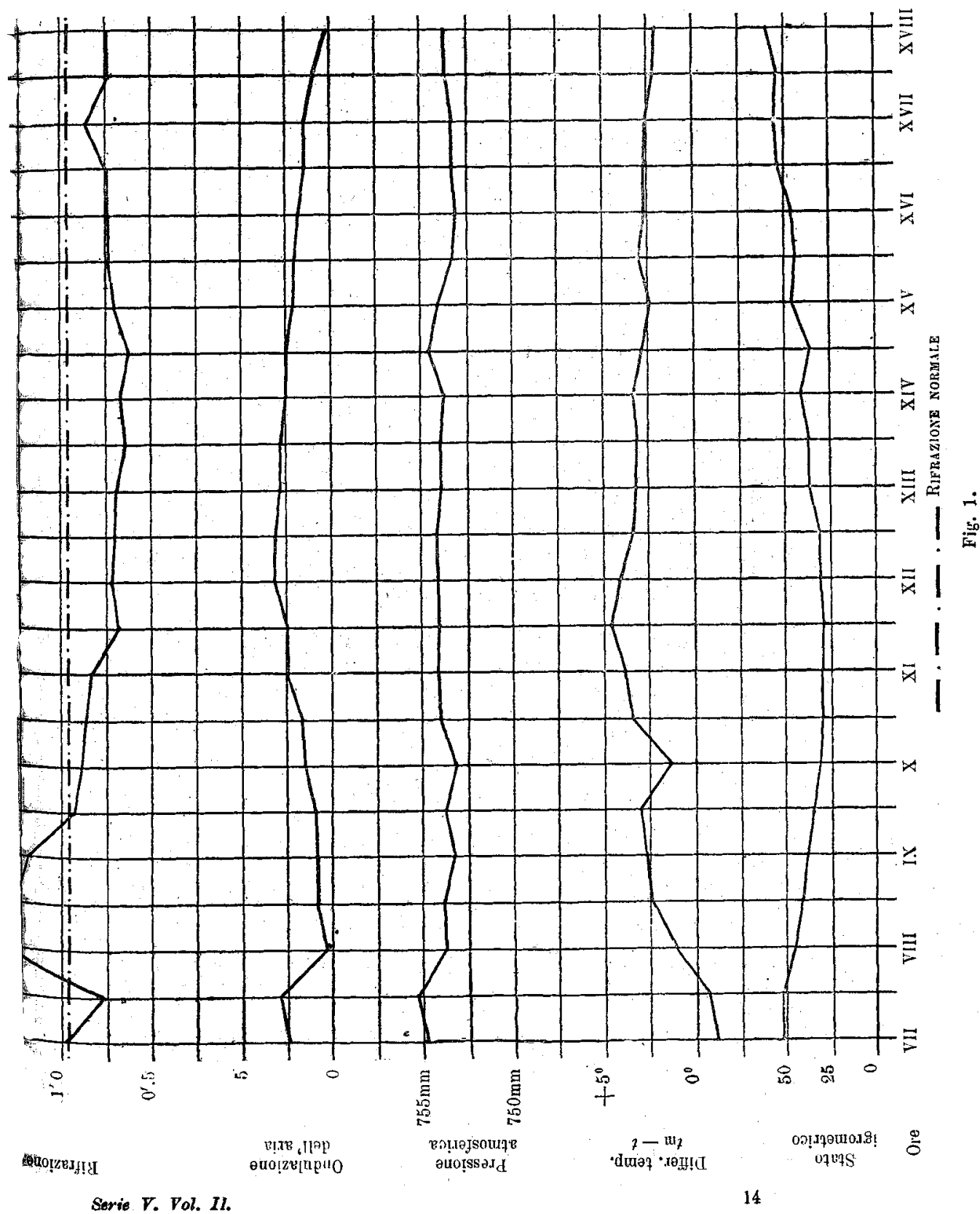


con altre osservazioni fatte insieme, determinato l'equazione personale di ciascuno.

Da quest' altro specchio (tabella II), nel quale riporto le medie delle depressioni osservate nelle ore antimeridiane e le medie delle depressioni osservate nelle ore pomeridiane, colle rispettive rifrazioni e lo stato del cielo di ogni singolo giorno di osservazione,

TABELlLA II.

\begin{tabular}{|c|c|c|c|c|c|c|c|c|}
\hline \multirow[b]{2}{*}{ Gioruo } & \multirow{2}{*}{$\begin{array}{c}\text { Stato } \\
\text { del cielo }\end{array}$} & \multicolumn{3}{|c|}{ Depressione media } & \multirow{2}{*}{$\mid \begin{array}{c}\text { Yarizizione } \\
\text { depress. } \\
d_{p}-d_{a}\end{array}$} & \multicolumn{3}{|c|}{ Rifrạzione media } \\
\hline & & $\left\{\begin{array}{c}\text { nelle ore } \\
\text { antimerid. } \\
d_{\mathbf{a}}\end{array}\right.$ & $\begin{array}{c}\text { nelle ore } \\
\text { pomerid. } \\
\left(d_{p}\right)\end{array}$ & diurna & & $\begin{array}{c}\text { nelle } \\
\text { ore } \\
\text { antimer. }\end{array}$ & $\begin{array}{c}\text { nelle } \\
\text { ore } \\
\text { pomerid. }\end{array}$ & diurna \\
\hline 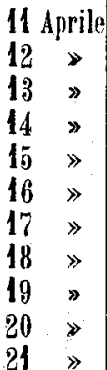 & $\begin{array}{c}\text { coperto } \\
\text { coperto } \\
\text { semi-rop. } \\
\text { sereno } \\
\text { coperto } \\
\text { sereno } \\
\text { sereno } \\
\text { coperto } \\
\text { sereno } \\
\text { sertuo } \\
\text { sereno }\end{array}$ & $\begin{array}{l}13^{\prime} .91 \\
12^{\prime} .48 \\
13^{\prime} .13 \\
14^{\prime} .68 \\
14^{\prime} .48 \\
13^{\prime} .70 \\
13^{\prime} .91 \\
14^{\prime} .52 \\
14^{\prime} .75 \\
15^{\prime} .00 \\
15^{\prime} .00\end{array}$ & $\begin{array}{c}14^{\prime} .16 \\
f \\
14^{\prime} .65 \\
13.95 \\
14^{\prime} .21 \\
14^{\prime} .65 \\
14^{\prime} .97 \\
15^{\prime} .19 \\
15^{\circ} .09\end{array}$ & $\begin{array}{l}14.06 \\
14.58 \\
14.86 \\
15.00 \\
15.04\end{array}$ & $\begin{array}{l}0^{\prime} .25 \\
0^{\prime} .30 \\
0^{\prime} .13 \\
0^{\prime} .22 \\
0^{\prime} .19 \\
0^{\prime} .09\end{array}$ & $\begin{array}{l}l^{\prime} .45 \\
2^{\prime} .88 \\
2^{\prime} .23 \\
0^{\prime} .68 \\
0.88 \\
l^{\prime} .66 \\
l^{\prime} .45 \\
0^{\prime} .84 \\
0^{\circ} .61 \\
0^{\prime} .36 \\
0^{\prime} .36\end{array}$ & $\begin{array}{c}1.20 \\
0.71 \\
0.71 \\
1.41 \\
1.15 \\
0.71 \\
0.39 \\
0.17 \\
0^{\circ} .27\end{array}$ & $\begin{array}{l}1.45 \\
2.04 \\
2.23 \\
0.70 \\
0.88 \\
0^{\prime} .53 \\
l^{\prime} .30 \\
0.78 \\
0.50 \\
0.27 \\
0.32\end{array}$ \\
\hline
\end{tabular}

emerge chiaramente che:

La depressione nelle ore antimeridiane (eccettuato il giorno 14 aprile in cui puó ritenersi costante) fu costantemente minore di quella delle ore pomeridiane, e per conseguenza la rifrazione fu maggi, re nelle ore antimeridiane che' nelle ore pomeridiane; la media delle rifrazioni avvenute con cielo sereno è minore della media delle rifrazioni avvenute con cielo coperto.

Quest' ultima conclusione, se ricevesse conferma da ulteriori osservazioni, mi sembra potrebbe essere una ragione per spiegare il fenomeno della minor ritrazione in Sicilia in confronto a quella osservata in altre regioni. Infatti è noto, che nel Meridionale tutto, ed in particolare in Sicilia, il cielo è 
più puro, più sereno che altrove e conseguentemente la rifrazione dovrà essere minore.

Avendo un discreto numero di misure di depressione posso prendendo la media di queste doterminare colla semplice formula

$$
n=\frac{2\left(d-d_{r}\right)}{d}
$$

il coeficiente di rifrazione marina $n$. Essendo $d=15,36$ $d_{r}$ medio $=14,53$ sarà

$$
n=0.1073
$$

valore poco discosto da quello ultimamente determinato nell'anno 1899 dal Prof. E. Soler a Palermo e dato da

$$
n=0,1026 \text {. }
$$

Dalla qual concordanza emerge che il metodo semplicissimo proposto dal Prof. Ricco per lo studio della variazione della rifrazione atmosferica si puó applicare per la determinazione del coefficiente di rifrazione marina in sostituzione di quelli complicati e difficoltosi adottati sino ad ora.

\section{IIBRI NUOVI}

\section{MANUALE DI BLETTROTECNIEA,}

\section{GRAWINKEL $e$ STREGKER.}

Edizigne italiana ampliata sulla sesta edizione tedesca, dall'ing FLAVIO DESSY.

(Ulrico Hoepli, Milano 1901)

E questo un manuale che merita di essere segnalato, come quello che racehiude, in uno spazio relativamente ristretto, i risultati di studi teorici e sperimentali di elettricità che si succedettero con rapidita meravigliosa, specialmente, in questi ultimi anni.

Esso non è un vero trattato di elettrotecnica, ma bensi un manuale pratico, interamente composto di nozioni generali, di 